\title{
1 Quantum Dynamics of Vortices and Vortex Qubits
}

\author{
A. Wallraff', A. Kemp, and A. V. Ustinov \\ Physikalisches Institut III \\ Universität Erlangen-Nürnberg \\ D-91058 Erlangen, Germany
}

\subsection{Introduction}

Vortices appear naturally in a wide range of gases and fluids, both on very large scales, e.g. when tornados form in the earths atmosphere, and on very small scales, e.g. in BoseEinstein condensates of dilute atomic gases [1] or in superfluid helium [2], where their existence is a consequence of the quantum nature of the liquid. Collective nonlinear excitations such as the vortex considered here are ubiquitous in solid state systems (e.g. domain walls), biological systems (e.g. waves on membranes) and have even been considered as model systems in particle physics. In superconductors, which we consider here, quantized vortices of the supercurrent [3], that are generated by magnetic flux penetrating into the material, play a key role in understanding the material properties [4] and the performance of superconductor-based devices $[5,6]$. At high temperatures the dynamics of vortices is essentially classical. At low temperatures, however, there are experiments suggesting the collective quantum dynamics of vortices [7, 8]. Here we report on experiments in which we have probed for the first time the quantum dynamics of an individual vortex in a superconductor. By measuring the statistics of the vortex escape from a controllable pinning potential we were able demonstrate the quantization of the vortex energy within the trapping potential well and the quantum tunnelling of the vortex through the pinning barrier.

The object that we have studied in our experiments is a vortex of electric current with a spatial extent of several tens of micrometers formed in a long superconducting tunnel junction. The electrodynamics of such a Josephson junction is governed by the phase difference $\varphi$ between the macroscopic wave functions describing the superconducting condensate in the two electrodes [9]. It is a well established fact that the variable $\varphi$ displays macroscopic quantum properties in point-like junctions at very low temperatures [10, 11]. Such macroscopic quantum phenomena are currently exploited for quantum information processing [12] using superconducting devices $[10,13,14,15,16,17,18,19,20]$. In extended one- or twodimensional Josephson junction systems quantum tunnelling in real space is to be expected for superconducting vortices, which are particle-like collective excitations of the phase difference $\varphi$. The small value of the expected mass of the vortex studied here suggests that quantum effects are likely to occur with vortices at low temperatures. Dissipative vortex tunnelling

\footnotetext{
${ }^{1}$ current address: Department of Applied Physics, Yale University, New Haven, CT 06520, USA; email: andreas.wallraff@yale.edu
} 
has been named a reason for the non-vanishing relaxation rate of the magnetization in type-II superconductors as the temperature $T$ is lowered towards zero. However, the quantum vortex creep model [8], which was suggested as an explanation for this behavior, faced orders of magnitude discrepancies with experimental data [21]. Alternative classical explanations have been suggested more recently to explain the low-temperature relaxation [22]. Nonetheless, macroscopic quantum tunnelling was observed for states with many vortices in discrete arrays of small Josephson junctions [23, 24]. For typical arrays the calculated vortex mass is about 500 times smaller than the electron mass [7]. All previous research in this area has focused on the collective behavior of a large number of vortices. Until now there had been no direct experimental observation of tunnelling events of individual vortices.

In Sec. 1.2 we discuss the experimental observation of quantum tunnelling and energy level quantization of an individual vortex. The formation and subsequent dissociation of vortex-antivortex pairs is covered in Sec. 1.3. In Sec. 1.4, the prospects of using quantum vortices in heart-shaped junctions as qubits for quantum information processing are evaluated and results on the manipulation of bistable vortex states are presented.

\subsection{Macroscopic quantum effects with single vortices}

Among many different vortex structures in superconductors, there is a rather special type of vortices in long Josephson junctions. These vortices have a unique character of solitons nonlinear waves that preserve their shape with time and propagate as ballistic particles [25]. These vortices are distinct from Abrikosov vortices in type-II superconductors, as they have no normal core and thus move with very low damping. In contrast to vortices in Josephson arrays [7], solitons in uniform long junctions do not generate any radiation during their motion and are well decoupled from other electromagnetic excitations in these systems. The quantum tunnelling of Josephson vortices in long junctions has been predicted theoretically [26, 27]. In our experiments we have observed this effect for the first time [28].

\subsubsection{Quantum tunnelling}

We have probed the quantum properties of a single Josephson vortex in a current-biased annular junction subject to an in-plane magnetic field $\vec{H}$, see inset of Fig. 1.1c. The junction of diameter $d=100 \mu \mathrm{m}$ and width $w=0.5 \mu \mathrm{m}$ is etched from a sputtered $\mathrm{Nb} / \mathrm{AlO}_{x} / \mathrm{Nb}$ thin film trilayer which is patterned using electron-beam lithography [29]. A photograph of the sample taken using an optical microscope is shown in Fig. 1.1a. Initially the vortex is topologically trapped in the junction by cooling the sample in a small perpendicular magnetic field $\vec{H}_{\text {tr }}$ which is generated using a separate pair of coils, the axis of which is perpendicular to the junction plane, see Fig. 1.1b. A single vortex in an annular junctions subject to an in-plane field behaves as a particle [30] in a tilted washboard potential [31,32]. The component of the potential periodic in the vortex coordinate $\Theta$ is due to the interaction $\vec{\mu} \cdot \vec{H} \propto \cos \Theta$ of the vortex magnetic moment $\vec{\mu}$ with the external magnetic field $\vec{H}$, see Fig. 1.1c. The tilt of the potential is proportional to the Lorentz force acting on the vortex which is induced by the bias current $I$ applied to the junction. The vortex may escape from a well in the tilted potential by a thermally activated process or by quantum tunnelling. At low temperatures 
(a)

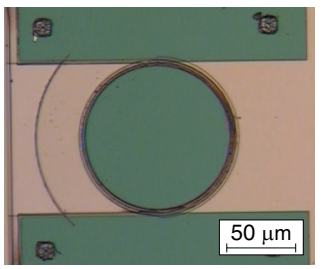

(b)

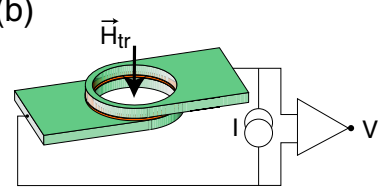

(c)

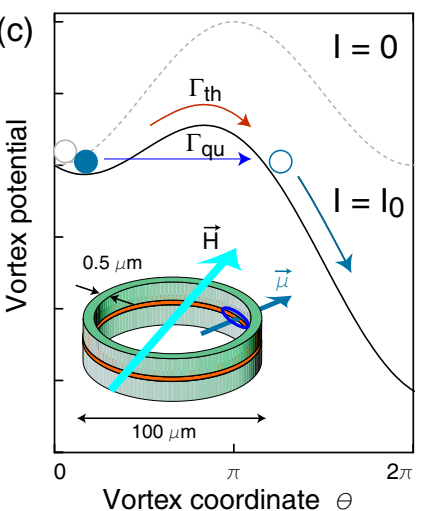

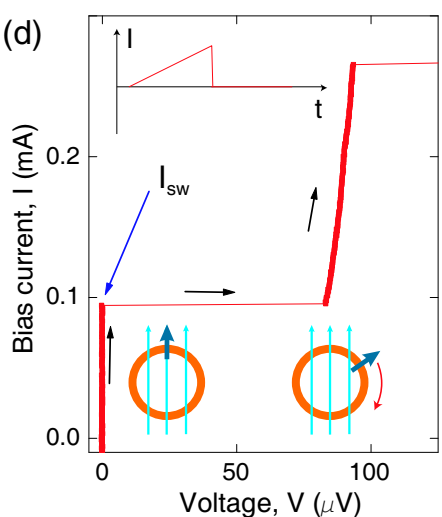

Figure 1.1: Sample, vortex potential and switching current measurement. (a) Photograph of the sample taken with an optical microscope. (b) Sketch of bias lead configuration and direction of trapping field $\vec{H}_{\text {tr. }}$. We employ a bias lead geometry which minimizes self field effects[36] and apply the magnetic field $\vec{H}$ generating the vortex potential in the plane of the junction but perpendicular to the bias leads. In this case the vortex is depinned in a location along the junction where self fields are minimal. (c) A vortex with magnetic moment $\vec{\mu}$ trapped in an annular Josephson junction subject to an in-plane external magnetic field $\vec{H}$. The junction width and diameter are indicated (see inset). The resulting vortex potential at zero bias current $I$ (dashed line) and at finite bias $I_{0}$ (solid line) is plotted versus the vortex coordinate $\Theta$ in the annulus. Different escape processes of the vortex are indicated. (d) Currentvoltage characteristic showing the vortex depinning from the field induced potential at a random value of bias $I_{\mathrm{sw}}$ when ramping up the bias current at a constant rate in a saw-tooth pattern (see upper inset). The transition of a pinned vortex state to a running vortex state is associated with a voltage appearing across the junction which is proportional to the vortex velocity (see lower insets). In experiment, the bias current is switched off immediately after a voltage is detected.

thermal activation is exponentially suppressed and the escape occurs by quantum tunnelling. This process is identified by measuring the temperature dependence of the distribution $P$ of depinning currents $I_{\mathrm{sw}}[33,34]$ of the vortex trapped in the junction. The $P\left(I_{\mathrm{sw}}\right)$ distributions are recorded by repeatedly ramping up the bias current at a constant rate $\dot{I}$ and recording the statistics of the current $I_{\mathrm{sw}}$ at which the vortex escapes from the well, which is associated with a switching of the junction from its zero-voltage state to a finite voltage state, see Fig. 1.1d. Our measurement technique and setup have been tested and calibrated $[34,35]$ in experiments on macroscopic quantum tunnelling of the phase in small Josephson junctions [11].

The bias current $I_{p}$ at which vortex tunnelling occurs with the highest probability (for a given bias current ramp rate) is found - as expected [31,32] - to be proportional to the applied magnetic field (data shown in Fig. 1.3c). This indicates, that the shape of the potential is controlled in our experiment by both field and bias current. The bias current induced self magnetic field effect on the vortex potential has been minimized by using an appropriate bias lead configuration and field direction, see Fig. 1.1b.

To search for quantum tunnelling of the vortex, the temperature dependence of the switching current distribution $P\left(I_{\mathrm{sw}}\right)$ has been measured. In Fig. 1.2a such distributions are shown for a magnetic field of $H=0.9$ Oe applied in the plane of the junction. It is clearly ob- 

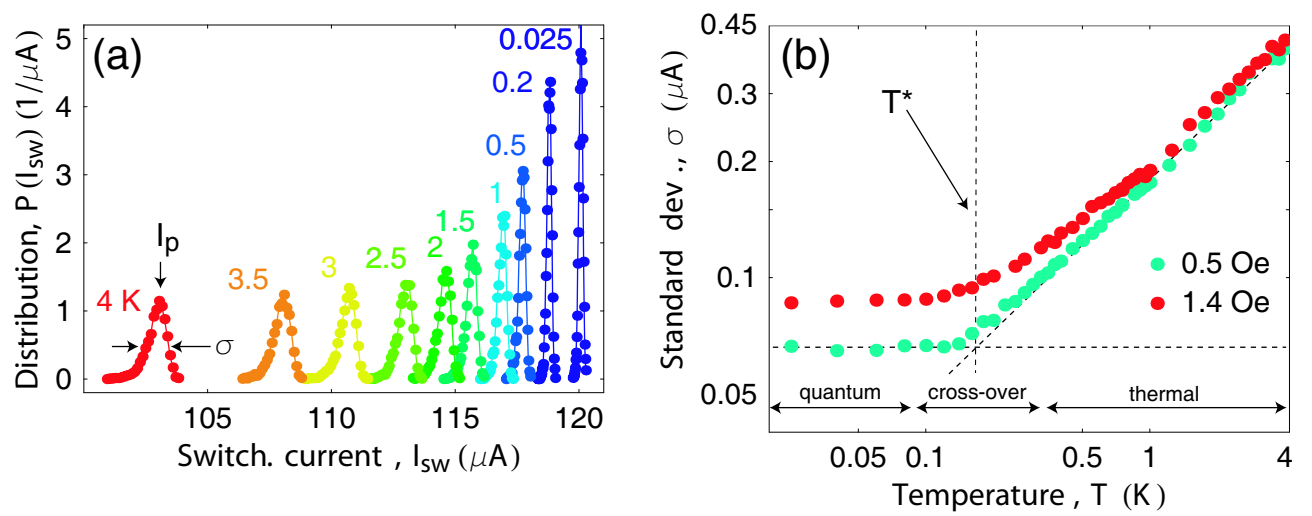

Figure 1.2: Thermal activation and quantum tunnelling. (a) Switching current distributions $P\left(I_{\mathrm{sw}}\right)$ at magnetic field $H=0.9$ Oe for bath temperatures $T$ between $4.0 \mathrm{~K}$ and $25 \mathrm{mK}$. (b) Standard deviation $\sigma$ of $P\left(I_{\mathrm{sw}}\right)$ versus $T$ for two values of field indicating the cross over in the vortex escape process from thermal activation to quantum tunnelling. The cross over temperature range around $T^{\star}$ is indicated. We have carefully verified experimentally that the saturation of the distribution width with temperature is not induced by excess noise or heating of the sample. The resolution of our measurement setup [34] is factor of 3 to 4 larger than the most narrow distribution widths measured in these experiments.

served that the distribution width $\sigma$ decreases with temperature and then saturates at low $T$. In Fig. $1.2 \mathrm{~b}, \sigma$ is plotted versus temperature on a double logarithmic scale for two different values of field. At high temperatures the distribution width is temperature dependent, indicating the thermally activated escape of the vortex from the well. In the high temperature limit $\sigma$ is in good approximation proportional to $T^{2 / 3}$ as expected for a thermally activated escape of a particle from a washboard potential close to critical bias. The distribution width $\sigma$ saturates at a temperature of about $100 \mathrm{mK}$. This behavior indicates the cross over of the vortex escape process from thermal activation to quantum tunnelling. At temperatures below $100 \mathrm{mK} \sigma$ is constant and the escape is dominated by quantum tunnelling. As expected, the cross-over temperature $T^{\star}$ is dependent on magnetic field, but only rather weakly. We attribute this observation to the fact that the vortex may change its shape when traversing the barrier during the escape process. This aspect can not be captured in the single particle model[30, 31] but rather is a consequence of the fact that the vortex is a collective excitation.

\subsubsection{Energy level quantization}

To probe the energy levels of the vortex in the potential well we have measured the vortex escape in the presence of microwave radiation using spectroscopic techniques which we have extensively tested on small Josephson junctions [35]. At low temperatures and in the absence of microwave radiation the vortex tunnels out of the ground state of the potential well into the continuum. The occupation of excited states is exponentially small, if the level separation is larger than the temperature. By irradiating the sample with microwaves the vortex can be excited resonantly from the ground state to the first excited state, see inset of Fig. 1.3b. In this case we observe a double peak structure in the switching current distribution, see Fig. 1.3a. 

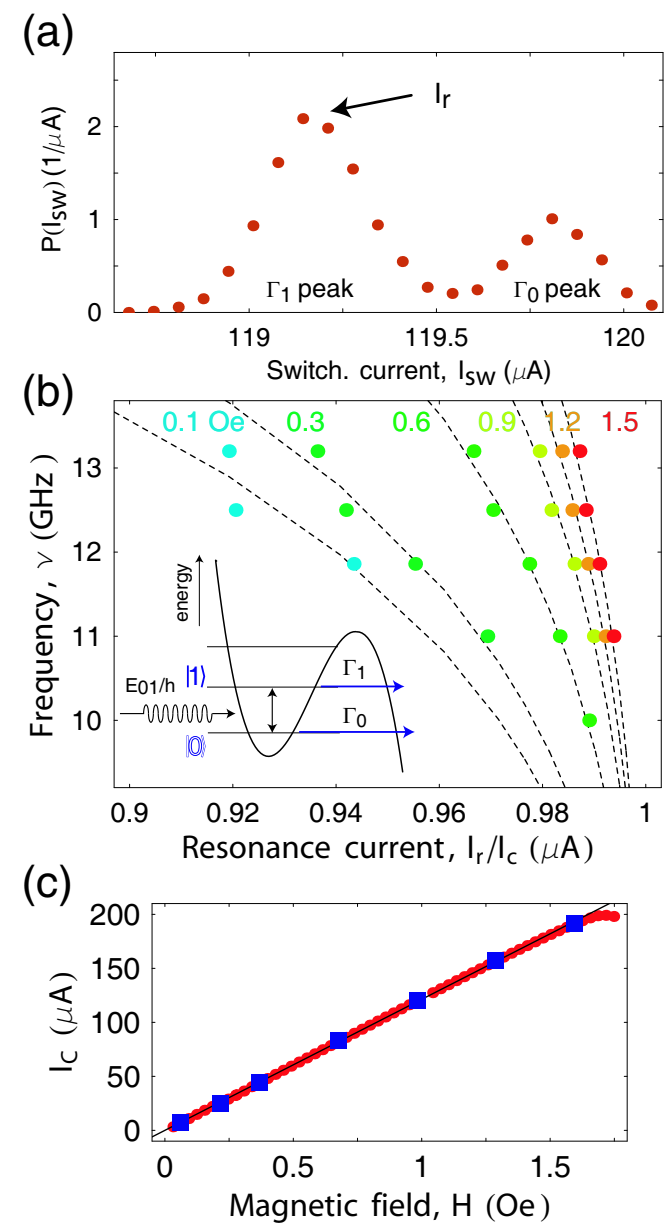

Figure 1.3: Vortex energy levels. (a) $P\left(I_{\mathrm{sw}}\right)$ distribution at $H=0.6$ Oe in the presence of microwave radiation of $\nu=11 \mathrm{GHz}$ at $T=25 \mathrm{mK}$. The resonance current $I_{r}$ at which tunnelling from the first excited state is most probable is indicated. (b) Microwave frequency $\nu$ versus normalized resonance current $I_{r} / I_{c}(H)$ for magnetic fields $H$ between 0.1 and 1.5 Oe. Dashed curves are fits to $\nu_{01}(H, I=$ $0)\left(1-\left(I / I_{c}(H)\right)^{2}\right)^{1 / 4}$. In the inset the tunnelling from the ground state and from the first excited state populated by resonant microwave radiation is indicated. (c) Critical current $I_{c}$ extracted from microwave spectroscopy (solid squares), most probable switching current $I_{p}$ at $T=25 \mathrm{mK}$ in absence of microwaves (solid circles) and fit (solid line) versus magnetic field $H$.

The peak at higher bias current is due to the tunnelling of the vortex from the ground state, whereas the peak at lower bias current corresponds to the tunnelling out of the first excited state.

The energy level separation scales both with bias current and applied magnetic field. To investigate this property, we have spectroscopically determined the separation between the ground and the first excited state by varying the microwave frequency and the magnetic 
field. For each value of the magnetic field we have determined the resonance current $I_{r}$ (see Fig. 1.3a) for a few different microwave frequencies $\nu$. In Fig. 1.3b, the applied microwave frequency $\nu$ is plotted versus the resonance current $I_{r}$ normalized by the depinning current $I_{c}(H)$ at that field in absence of microwaves and fluctuations. It is observed that all data points show the characteristic scaling of the energy level separation $\Delta E_{01} / h$ with the bias current as $\nu_{01}(H, I)=\nu_{01}(H, I=0)\left(1-\left(I / I_{c}(H)\right)^{2}\right)^{1 / 4}$ as expected for the tilted washboard potential. The data at each field are fitted to this dependence using the characteristic frequency $\nu_{01}(H, I=0)$ of vortex oscillations at zero current and the depinning current $I_{c}(H)$ as fitting parameters, see dashed lines in Fig. 1.3b. The resonance frequency $\nu_{01}(H, I=0)$ and the depinning current $I_{c}(H)$ in absence of fluctuations have been determined from the fit. As expected, the energy level separation $\Delta E_{01}$ increases with field and the depinning current $I_{c}$ as determined from the spectroscopic data is linear in the field, see Fig. 1.3c, which is in excellent agreement with the current $I_{p}$ measured directly in absence of microwaves.

From the resonance frequency at a given bias current we can estimate the cross-over temperature, which in the limit of small damping is given by $T^{\star} \simeq h \nu_{01}(H, I) / 2 \pi k_{B}$. Thus we can compare the cross-over temperature extracted from the temperature dependence of the switching current distributions to the predictions based on the data extracted from spectroscopic measurements. For $\nu_{01}(H, I)$ between 10 and $13 \mathrm{GHz}$ we find a value of $T^{\star}$ between approximately 75 and $100 \mathrm{mK}$, which is consistent with the measured saturation temperature in Fig. 1.2.

\subsection{Vortex-antivortex pairs}

The thermal and the quantum dissociation of a single vortex-antivortex (VAV) pair in an annular Josephson junction is experimentally observed and theoretically analyzed. In our experiments the VAV pair is confined in a pinning potential controlled by external magnetic field and bias current. The dissociation of the pinned VAV pair manifests itself in a switching of the Josephson junction from the superconducting to the resistive state. The observed temperature and field dependence of the switching current distribution is in agreement with the analysis. The crossover from the thermal to the macroscopic quantum tunneling mechanism of dissociation occurs at a temperature of about $100 \mathrm{mK}$.

\subsubsection{Thermal and quantum dissociation}

In this section we report on the experimental observation of thermal and the quantum dissociation of a single vortex-antivortex pair [37]. States containing many VAV pairs are relevant to thin superconducting films or large two-dimensional Josephson arrays close to the KosterlitzThouless transition [9]. A single VAV pair naturally appears in a long annular JJ placed in an external magnetic field $H$ parallel to the junction plane [31,38] (Fig. 1.4a-b). For experiments we fabricated a junction of diameter $d=100 \mu \mathrm{m}$ and width $w=0.5 \mu \mathrm{m}$ which was etched from a sputtered $\mathrm{Nb} / \mathrm{AlO}_{x} / \mathrm{Nb}$ thin film trilayer and patterned using electron-beam lithography [29]. Its critical current density is $220 \mathrm{~A} / \mathrm{cm}^{2}$, the Josephson length is $\lambda_{J} \approx 30 \mu \mathrm{m}$ and the normalized junction length is $L \equiv \pi d / \lambda_{J} \approx 10.5$. The measured magnetic field dependence of the switching current is shown in Fig. 1.4c. In the field range $|H|<1.5$ Oe (main central 
lobe), the switching of the $\mathrm{JJ}$ from the superconducting state to the resistive one occurs through the dissociation of a single field-induced VAV pair confined in the potential well created by externally applied magnetic field and dc bias current. This process is confirmed by direct numerical simulations of the full sine-Gordon equation for an annular JJ [31, 38] of length $L$. The numerically found magnetic field dependence of the switching current is in excellent agreement with the measurement, see solid line in Fig. 1.4c. Simulations of the magnetic field distribution in the junction clearly show the nucleation and subsequent dissociation of the VAV pair, see Fig. 1.4d. Fluctuations, thermal and quantum, induce internal oscillations of the confined pair. At high temperature the dissociation then takes place by thermal activation over the barrier. At low temperature macroscopic quantum tunneling through the barrier occurs. At fields $|H|>1.5$ Oe the system becomes bistable as a well-separated VAV pair penetrates in the junction. This state is perfectly reproduced by our numerical calculations, see the first side lobes in Fig. 1.4c, and its quantum dynamics will be discussed elsewhere.

We have theoretically analyzed the penetration and the following dissociation of a VAV pair in the presence of a small magnetic field $H$ and a large dc bias current $I$ [37]. The bound vortex-antivortex pair is confined by a potential formed by the bias current and the magnetic field. Its dissociation can be mapped into the well known problem of particle escape from a cubic potential [37]. The probability of the dissociation depends on the height of the effective potential barrier

$$
U_{\text {eff }}(\delta)=2 \cdot 3^{5 / 4} h^{-1 / 4}\left(\delta-\delta_{c}(h)\right)^{3 / 2},
$$

where $\delta=\left(I_{c 0}-I\right) / I_{c 0} \ll 1$, with $I_{c 0}$ being the critical current of a long $\mathrm{JJ}$ for $H=0$, $h \propto H$ is the normalized external magnetic field, and $\delta_{c}(h)=2 h / 3$ is the critical current in the absence of fluctuations.

At high temperatures, the dissociation is driven by thermal activation over this barrier. Using the known theory describing the escape from such a potential well $[9,11]$, we find the switching rate of a long Josephson junction from the superconducting state to the resistive state

$$
\Gamma_{T}(I) \propto \exp \left[-U_{\mathrm{eff}} / k_{B} T\right]
$$

Thus, at high temperature the standard deviation of the critical current $\sigma$ increases with temperature and weakly depends on the magnetic field: $\sigma_{T} \propto T^{2 / 3} h^{1 / 6}$. Notice that $\sigma_{T}$ increases with $H$, in contrast to the behavior of a small Josephson junction $[9,11]$ where $\sigma_{T} \propto\left(I_{c}(H)\right)^{1 / 3}$ decreases with $H$.

We have experimentally investigated the fluctuation induced dissociation of the VAV pair by measuring the temperature and magnetic field dependence of the statistical distribution $P$ of the switching currents $I<I_{c 0}$ using techniques described in [34]. In Fig. 1.5a the temperature dependence of the switching current distribution measured at $H=0$ is shown. At high temperatures the $P(I)$ distribution is temperature dependent, at low temperatures a saturation is observed. In Fig. 1.5b, the standard deviation $\sigma$ of $P(I)$ is plotted versus bath temperature $T$ for two values of magnetic field. $\sigma$ is well approximated by $T^{2 / 3}$ dependence on the temperature, and the standard deviation is larger for the higher field as predicted in the above analysis. As clearly seen in Fig. 1.5b, $\sigma$ decreases with temperature and saturates below a cross-over temperature of $T^{\star} \approx 100 \mathrm{mK}$. At $T<T^{\star}$ the dissociation of the VAV 
(a)

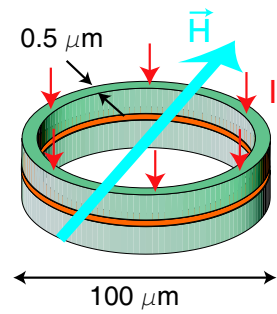

(c)

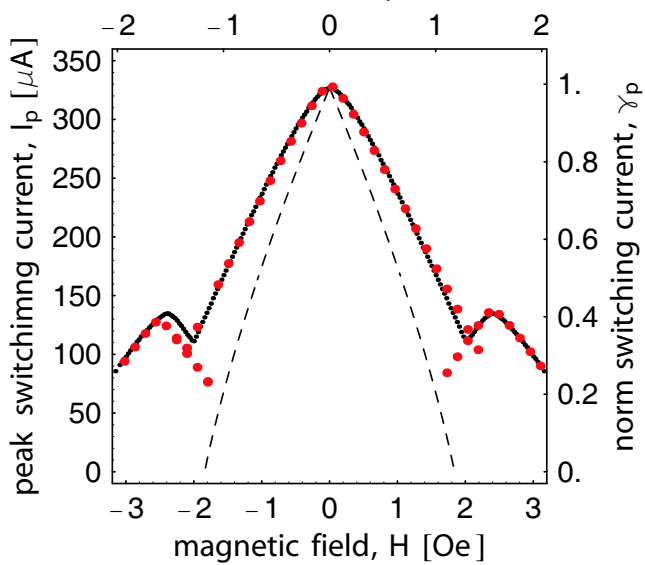

(b)

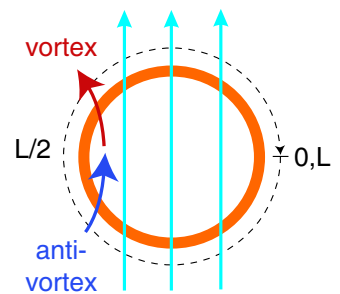

(d)

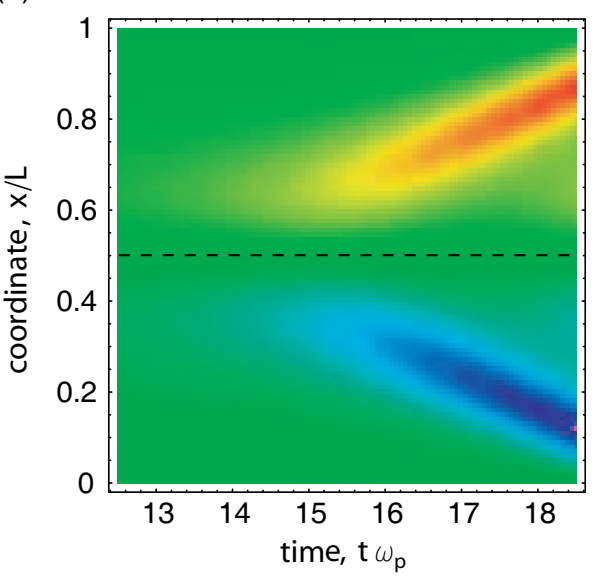

Figure 1.4: (a) Schematic view of a long annular Josephson junction without trapped vortices in an in-plane external magnetic field $H$ with uniform bias current $I$. (b) Generation of a confined vortexantivortex pair with the center coordinate at $x=L / 2$. (c) Magnetic field dependence of switching currents: experimental data at $T=100 \mathrm{mK}$ (circles), numerical calculation for $L=10.5$ (solid line), and theoretical prediction of Eq. (1.6) (dashed line). (d) Numerically simulated evolution of the magnetic field distribution, as the Josephson junction switches into the resistive state. The emerging vortex and antivortex move in opposite directions.

pair occurs through a macroscopic quantum tunneling process. The cross-over temperature $T^{\star} \simeq \hbar \omega(\delta) /\left(2 \pi k_{B}\right)$ is determined by the frequency $\omega(\delta)$ of small oscillations of the VAV pair. In the quantum regime the frequency $\omega(\delta)=3^{3 / 8} / \sqrt{\chi}\left(\delta-\delta_{c}(h)\right)^{1 / 4}$ determines the oscillatory energy levels $E_{n} \simeq \hbar \omega(\delta)(n+1 / 2)$ of the pinned VAV state. We examined these levels experimentally by performing microwave spectroscopy [42] as demonstrated earlier for the case of a single Josephson vortex trapped in a long Josephson junction [28] and summarize the results in the subsection below.

Neglecting dissipative effects, in the quantum regime the switching rate $\Gamma_{Q}(I)$ of the under-barrier dissociation can be estimated, as usual, in the WKB approximation, which yields

$$
\Gamma_{Q}(I) \propto \exp \left(-\frac{36 U_{\mathrm{eff}}(\delta)}{5 \hbar \omega(\delta)}\right)
$$



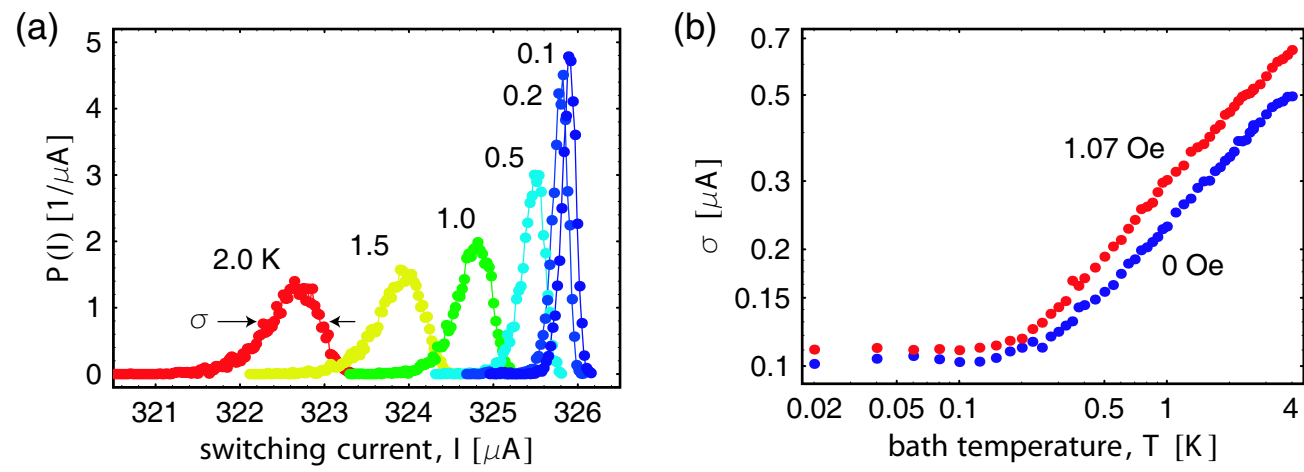

Figure 1.5: (a) Switching current distributions $P(I)$ at $H=0$ shown at different bath temperatures $T$. (b) Standard deviation $\sigma$ of $P(I)$ distributions versus bath temperature for the two indicated values of magnetic field.

In this limit the standard deviation of the critical current $\sigma$ is independent of temperature and (similar to the high temperature case), it weakly increases with magnetic field.

The above analysis is based on an assumption that the pair's size is small with respect to the JJ length. We find that the Josephson phase escape in the form of the dissociation of the pair occurs (in normalized units) as $h \geq \frac{3}{4}(L / 2)^{-4}$. In the opposite limit of very small magnetic field $h \lesssim(L / 2)^{-4}$ the Josephson phase escape occurs homogeneously in the whole junction $^{2}$.

The analysis presented above is valid for small magnetic fields, $h \ll 1$. As the magnetic field $h$ increases, the critical current $I_{c}(h)$ is suppressed, and only a qualitative description of the VAV pair dissociation can be carried out. In the general case the pair size is $l_{p} \simeq$ $\left(1-\left(I / I_{c 0}\right)^{2}\right)^{-1 / 4}$ (instead of $l_{p} \simeq \delta^{-1 / 4}$ that is valid for $h \ll 1$ ), and the amplitude of the state is $\xi_{p} \simeq \arccos \left(I / I_{c 0}\right)$ (instead of $\xi_{p} \simeq \sqrt{ } \bar{\delta}$ ). Following a similar procedure as in Ref. [37] we obtain the standard deviation dominated by the thermal fluctuations

$$
\sigma_{T} \simeq \frac{T^{2 / 3} h^{2 / 3}}{\arccos \left(\frac{I_{c}(h)}{I_{c 0}}\right)},
$$

and in the quantum regime

$$
\sigma_{Q} \simeq \frac{h}{\left(\arccos \frac{I_{c}(h)}{I_{c 0}}\right)^{8 / 5}} .
$$

The standard deviation is determined by the magnetic field dependence of the critical current $I_{c}(h)$ that is given implicitly by the equation

$$
h=\frac{3}{4} \sqrt{\left(1-\left(I_{c}(h) / I_{c 0}\right)^{2}\right)} \arccos \left(\frac{I_{c}(h)}{I_{c 0}}\right) .
$$

\footnotetext{
${ }^{2}$ We do not consider here very long Josephson junctions where even in the absence of magnetic field fluctuationinduced vortex-antivortex pairs are generated in the Josephson junction [39, 40, 41].
} 


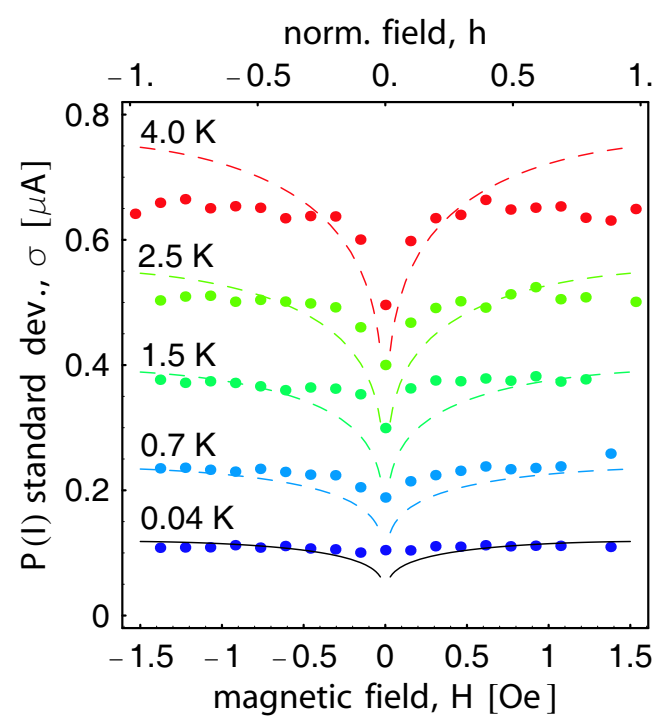

Figure 1.6: Standard deviation $\sigma$ of $P(I)$ distribution versus magnetic field $H$ in the temperature range between $40 \mathrm{mK}$ and $4.0 \mathrm{~K}$ : experiment (dots), theory [dashed lines, Eqs. (1.4) and (1.5)]. The region of magnetic field corresponds to the central lobe in the $I_{c}(H)$ dependence displayed in Fig. 1d.

This dependence is shown in Fig. 1d by a dashed line. The discrepancy between analysis and numerics in the values of $I_{c}$ is a consequence of the fact that the analysis has been carried out for junctions with length $L / 2 \pi \gg 1$ but the experimentally investigated system only barely meets that limit $(L \approx 10.5)$. However, the analytical predictions and numerics are in good accord as the length of the Josephson junction is increased to $L \geq 20$ (data are not shown).

In Fig. 1.6, the measured field dependence of the switching current distribution width $\sigma$ is shown for temperatures ranging from $40 \mathrm{mK}$ to $4 \mathrm{~K}$. The calculated dependencies $\sigma_{T / Q}(h)$ are shown in the same figure by dashed lines. At each temperature the distribution width $\sigma$ has a minimum, pronounced in the thermal regime, at zero magnetic field. In qualitative agreement with the analysis given by Eqs. (1.4) and (1.5), $\sigma$ shows an increase and a following saturation with magnetic field in both thermal and quantum regimes. This behavior is characteristic for the fluctuation induced dissociation of a VAV pair.

\subsubsection{Energy levels of a bound vortex-antivortex pair}

As in the single vortex case, the energy levels can be probed spectroscopically. The model in [37] predicts the small oscillation frequency to be given by

$$
\nu(I, H)=\nu_{0}(H)\left(1-\frac{I}{I_{c}(H)}\right)^{1 / 4} .
$$




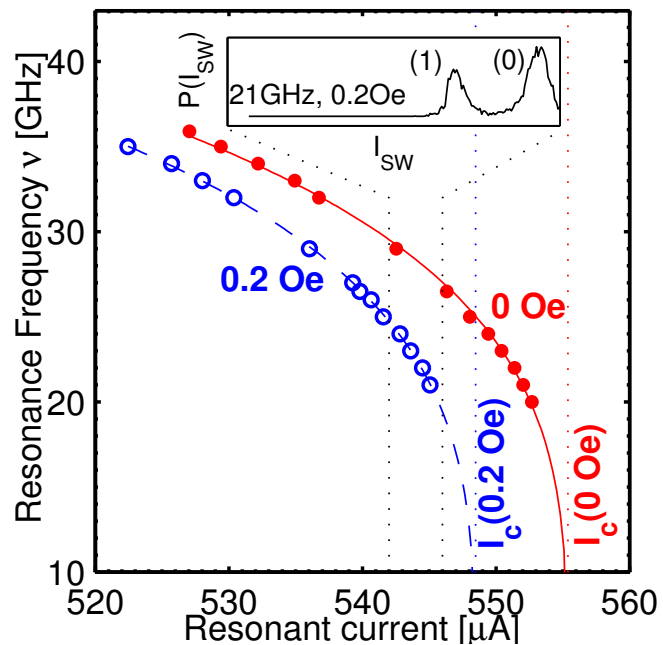

Figure 1.7: Resonance current versus frequency. Experimental data for zero magnetic field (filled circles) and 0.2 Oe (open circles) are compared to the model (solid line and dashed line, respectively). The respective critical currents are indicated by the dotted vertical lines. In the inset, the original switching-current probability distribution $P\left(I_{\mathrm{sw}}\right)$ is indicated for a single data point using an enlarged current scale. The peak (1) corresponds to escape from the resonant level, the peak (0) to escape from the ground state.

Here the scaling of $\nu(I, H)$ with $I / I_{c}(H)$ is the same as for the vortex or small junction case at a constant value of $H$ and $I / I_{c}(H)$ close to unity. The factor $\nu_{0}(H)$ is the magnetic-fielddependent internal oscillation frequency of the VAV pair at zero bias current. For a number of different values of magnetic field, we have measured the dependence of the resonance current on microwave frequency.

Experimental data for zero magnetic field and $H=0.2 \mathrm{Oe}$, which compare well to Eq. (1.7), are displayed in Fig. 1.7. Least-squares fits to the data for different magnetic fields yield both $I_{c}(H)$ and $\nu_{0}(H)$.

The theoretical dependence of $\nu_{0}(H)$ on $I_{c}(H)$ for VAV dissociation is modelled by [37]

$$
\nu_{0}(H)=\frac{\omega_{0}}{2 \pi} \frac{3^{3 / 8}}{\sqrt{\chi}}\left(\frac{I_{c}(H)}{I_{c 0}}\right)^{1 / 4},
$$

where $\omega_{0} / 2 \pi$ is the plasma frequency of the junction at $H=0$. This expression differs from that expected for phase escape in a small Josephson junction, where $\nu_{0}(H) \propto \sqrt{I_{c}(H) / I_{c 0}}$. In Fig. 1.8 we fit the experimentally determined magnetic field dependence of the zero bias oscillation frequency $\nu_{0}(H)$ to Eq. (1.8). For comparison, the expected oscillation frequency for homogeneous, small junction like escape of the phase is shown by the dotted line in the same plot. We note that the field dependence of the zero bias oscillation frequency extracted from experimental data is in good agreement with the predictions based on our model for 


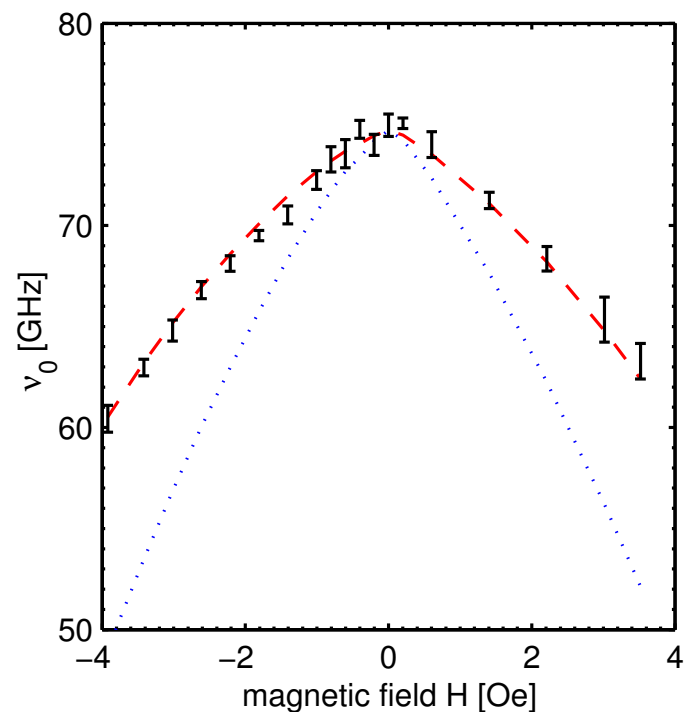

Figure 1.8: Field dependence of zero bias oscillation frequency $\nu_{0}$ extracted form measured data (solid error bars), $\nu_{0}(H)$ calculated from $I_{c}(h)$ using the long-junction model (dashed line) and the smalljunction model (dotted line).

VAV dissociation, while the prediction for the homogeneous phase escape clearly disagrees with the experimental data.

\subsection{The Josephson Vortex Qubit}

In this section we discuss the design of a qubit based on a single Josephson vortex trapped in a shaped long Josephson junction. The vortex potential is formed due to its interaction with an in-plane magnetic field and a bias current applied to the junction. The profile of the potential is calculated using a standard perturbation approach. We examine the dependence of the potential properties on the junction shape and its electrical parameters and discuss the requirements for observing quantum effects in this system. We have developed and experimentally tested methods for the preparation and read-out of vortex states of this qubit in the classical regime.

In the past years several types of different superconducting circuits [13, 14, 15, 43, 44, 45] based on small Josephson junctions in the phase or charge regime have been shown to achieve parameters which are favorable for quantum computation. In Ref. [46], a qubit based on the motion of a Josephson vortex in a long Josephson junction was proposed. A major difference from the small Josephson junction qubit proposals where the effective potential is created by the Josephson or charging energy, is that in the vortex qubit the potential is formed by the magnetic interaction of the vortex magnetic moment with an external magnetic field, as described in Ref. [31].

In heart-shaped annular junctions two classically stable vortex states can be arranged, corresponding to two minima of the potential. While the external field is always applied in 
the plane of the long junction, its angle $\Theta$ and strength $h$ can be varied. The bias current across the junction can be used to tilt the potential. These parameters allow to manipulate and control the potential and to read out the qubit state using a depinning (zero-voltage) current measurement. The scheme of readout and preparation of the state for this type of qubit was already demonstrated in the classical regime as briefly described in Ref. [47].

Here we describe how an effective double well potential for the vortex can be constructed and determine the parameter range required for reaching the quantum regime. A scheme to implement elementary single-bit quantum gates using the two in-plane magnetic field components is presented. The single-vortex potential is calculated using a perturbation theory approach [30] and tunnelling rates in the quantum regime are determined by numerical diagonalization of the Hamiltonian and compared to a calculation in WKB approximation.

\subsubsection{Principle of the vortex qubit}

Josephson junctions, which have a length significantly larger and a width $w$ smaller than the Josephson penetration depth $\lambda_{J}$ are called long junctions. These junctions are described by the phase difference $\varphi(q)$ of the two superconductors as the continuous degree of freedom along the spatial coordinate $q$ (normalized to $\lambda_{J}$ ), where $0<q<l$, where $l$ is the length of the junction normalized to $\lambda_{J}$. A magnetic field threading the junction corresponds to a gradient in the phase difference along the junction. An electrical field across the junction corresponds to a time derivative of $\varphi$. The dynamics of a long Josephson junction is governed by the perturbed sine-Gordon equation as discussed below.

Our experiments deal with long annular junctions. These consist of two stacked superconducting rings separated by a tunnel barrier. Since the flux in every ring is quantized, it is possible to realize a situation, in which the difference between the fluxes in two rings is one flux quantum $\Phi_{0}$. In this case a vortex of supercurrent carrying this flux quantum is formed along the junction. A resting vortex confines the magnetic flux to a characteristic size of $\lambda_{J}$. Since a moving vortex corresponds to moving magnetic flux, a voltage proportional to the speed of the vortex appears across the junction and can be detected. The electrical energy stored in the system is proportional to the square of the average voltage drop across the junction. It corresponds to the kinetic energy of a moving particle. It is therefore possible to consider a vortex as a quasiparticle moving in one dimension.

The junction can be biased by a current across it. A Lorentz-type driving force is exerted by the bias current on the vortex. The vortex magnetic moment also interacts with the external field by a magnetic dipole interaction. This yields a possibility to create a potential for the vortex. The magnetic moment is always directed normal to the junction. By varying the angle of the junction centerline it is possible to change the potential energy of the vortex as it moves along the junction.

Using a geometry, which is shown in Fig. 1.9a, it is possible to generate a double-well potential for a vortex in the junction. We chose a shape, which consists of a semi-circle of radius $R$, and two connected arcs, which intersect each other at an angle of $2 \beta$. An external magnetic field $h$ is applied, at an angle $\Theta$. The field can be described by the components $h_{x}=h \sin \Theta$ and $h_{y}=h \cos \Theta$.

Assuming the vortex to carry a point-like magnetic moment, its stable positions of minimal magnetic energy can be easily found to be the regions, where the junction is aligned 
(a)

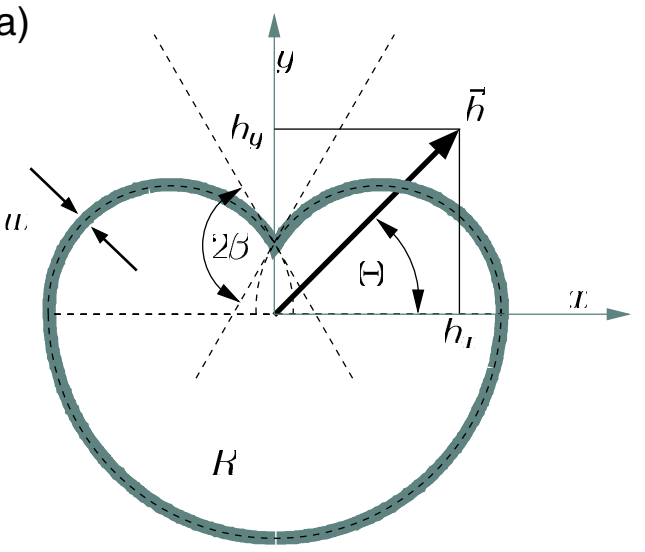

(b)

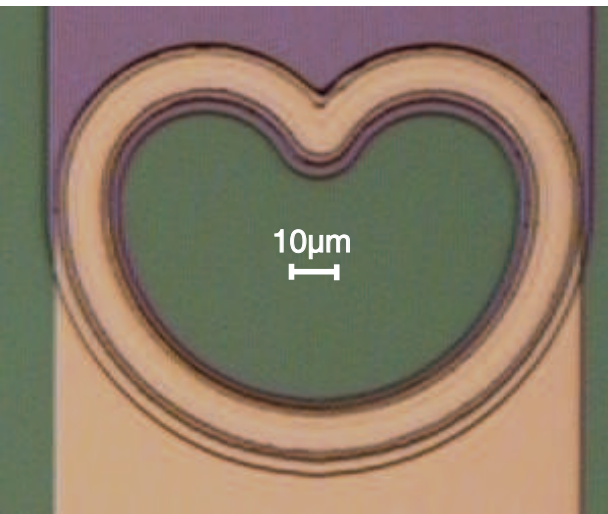

Figure 1.9: a) The geometry of the heart-shaped junction, defined by the two geometrical parameters $\beta$ and $R$. b) Optical microscope picture of the junction used for the experimental test. Parameters are $R=50 \mu \mathrm{m}, \beta=60^{\circ}, w=3 \mu \mathrm{m}, j_{C}=796 \mathrm{~A} / \mathrm{cm}^{2}$.

perpendicular to the field. In reality, the vortex is distributed over a length on the order of $\lambda_{J}$. This changes the potential shape considerably, and may even change its qualitative features. In the case of the double-well potential in the heart-shaped junction it may cause the barrier, which separates the two minima, to vanish.

Here we derive the exact effective potential in order to determine the range of parameters and geometries suitable for experiments in the thermal and quantum regime. We show that the effective potential for a vortex inside a shaped junction is a sum of three terms, which depend on the external bias current, and the two in-plane field components. These are the three parameters, which can be controlled during the experiment in order to realize degenerate bistable vortex states, change the barrier between them, lift the degeneracy in a controlled way, and, finally, read-out the state using a critical current measurement. Based on this approach we proceed to quantum mechanical calculations and the calculation of the depinning current.

\subsubsection{Model}

Classically, the evolution of the phase difference $\varphi$ between the wave functions of the two superconducting electrodes forming a long Josephson junction is described by the sine-Gordon equation

$$
\sin (\varphi)=\varphi_{q q}-\varphi_{t t},
$$

where the temporal coordinate is denoted by $t$, normalized to the plasma frequency $\omega_{P}^{-1}$, discussed below. Subscripts denote partial derivatives.

Adding the terms for the inductive energy, the capacitive energy and the Josephson energy 
yields the corresponding hamiltonian

$$
H=\int_{0}^{l}\left(\frac{1}{2} \varphi_{q}^{2}+\frac{1}{2} \varphi_{t}^{2}+1-\cos \varphi\right) \mathrm{d} q
$$

with the characteristic energy scale $E_{0}$, discussed below. The temporal derivative $\varphi_{t}$ corresponds to the normalized voltage across the junction, the spatial derivative $\varphi_{q}$ corresponds to the normalized magnetic field in the junction.

Single-vortex solutions of Eq. (1.9) in a non relativistic approximation for infinitely long junctions are given by

$$
\varphi\left(q, q_{0}\right)^{\text {vortex }}= \pm \arctan \left(\exp \left(q-q_{0}-v t\right)\right)
$$

where $q_{0}$ denotes the vortex center of mass and $v$ its velocity normalized to the Swihart velocity $\bar{c}$, which is the characteristic velocity for the electromagnetic waves in the junction.

In the case of weak magnetic field and small bias current, the interaction with field and current can be modeled using perturbation theory. In Ref. [30], a bias current $\gamma$ (normalized to the critical current) was found to exert a driving force of $2 \pi \gamma$ on the vortex. The influence of the external magnetic field on a shaped junction was studied theoretically [31] and experimentally [48] for a circular shape of the annular junction. The sine-Gordon equation was discussed previously for annular junctions of small $\left(2 \pi r<\lambda_{\mathrm{J}}\right)$ and large radius $\left(2 \pi r>\lambda_{\mathrm{J}}\right)$ in [38]. We use the overlap geometry, which is known to generate very little self field of the bias current. In [49] we determined experimentally the self-field effect for circular geometries corresponding to the heart-shaped junctions by measuring the dependence of the critical current on the angle of the external magnetic field. Since during the operation of the qubit no current is flowing, we made no attempt to treat the self-field quantitatively. Before we further investigate the more complex junction shapes, we define the characteristic scales and normalizations.

In a long Josephson junction there are three important scales, which characterize the classical and quantum dynamics of the unperturbed system. The Josephson length

$$
\lambda_{\mathrm{J}}=\frac{\Phi_{0}}{2 \pi} \sqrt{\frac{1}{L^{*} e_{\mathrm{J}}}}
$$

is determined by the inductance $L^{*}$ and the Josephson coupling energy $e_{J}$ per unit length in the junction. The length $\lambda_{\mathrm{J}}$ is the characteristic lateral dimension of Josephson vortex at rest. The Josephson coupling energy is related to the critical current density $j_{\mathrm{C}}$ as $e_{J}=j_{\mathrm{C}} \Phi_{0} / 2 \pi w$, where $w$ denotes the width of the long Josephson junction.

Small amplitude linear wave solutions of the phase are described by a dispersion relation. At the wave number $k=0$ (a homogeneous oscillation over the whole junction) the corresponding frequency is the so-called Josepshon plasma frequency $\omega_{P}$, given by

$$
\omega_{\mathrm{P}}=\frac{2 \pi}{\Phi_{0}} \sqrt{\frac{e_{\mathrm{J}}}{C^{*}}},
$$

where $C^{*}$ denotes the capacitance of the junction per unit length. The temporal coordinate of Eq. (1.9) is normalized by $\omega_{P}^{-1}$. The product $\lambda_{\mathrm{J}} \omega_{P}=\bar{c}$ is the Swihart velocity. All energies 
are normalized to the characteristic energy

$$
E_{0}=\frac{\Phi_{0}}{2 \pi} \sqrt{\frac{e_{\mathrm{J}}}{L^{*}}} w=e_{\mathrm{J}} \lambda_{J}
$$

The energy unit $E_{0}$ is equal to the Josephson coupling energy of a small Josephson junction of the area $\lambda_{\mathrm{J}} w$. The rest energy of a vortex is equal to 8 in units of $E_{0}$, where half the energy is stored in the Josephson coupling and the other half is stored in the inductive energy. Since the speed of light equals unity in the normalized units, the rest mass of the vortex $m_{0}=8$.

Applying these normalizations to the Planck's constant $\hbar$, which has the unit of action, yields a normalized Planck's constant $\hbar_{\text {norm }}$, given by

$$
\hbar_{\mathrm{norm}}=\hbar \frac{\omega_{p}}{E_{0}}=\hbar\left(\frac{2 \pi}{\Phi_{0}}\right) \sqrt{\frac{L^{*}}{C^{*}}} \frac{1}{w} .
$$

The normalized Planck's constant $\hbar_{\text {norm }}$ does not depend directly on the Josephson coupling energy, but $L^{*}, C^{*}$ and $e_{J}$ are related to each other through the barrier thickness.

\subsubsection{Perturbative calculation of vortex potential}

We apply a perturbation theory approach similar to that of Ref. [31] and Ref. [30] and reduce the dynamics of the system to the center of mass motion of the vortex as with its coordinate $q_{0}$ being the only degree of freedom. In the lowest order of perturbation theory it is assumed that the phase gradient profile imposed by the external magnetic field and the phase gradient profile corresponding to a resting vortex do not influence each other, which requires that at least one of these is assumed to be small. The inductive energy term of Eq. (1.10) for $\varphi_{q}=\varphi_{q}^{\text {vortex }}+\varphi_{q}^{\text {ext }}$ yields

$$
U^{\text {ext }}\left(q_{0}\right)=\int_{0}^{l} \frac{1}{2}\left[\varphi_{q}^{\text {vortex }}\left(q-q_{0}\right)+\varphi_{q}^{\text {ext }}(q)\right]^{2} \mathrm{~d} q .
$$

Expanding Eq. (1.16) yields

$$
\begin{aligned}
U^{\text {ext }}\left(q_{0}\right)= & \int_{0}^{l} \frac{1}{2} \varphi_{q}^{\text {vortex }}\left(q-q_{0}\right)^{2} \mathrm{~d} q+\int_{0}^{l} \frac{1}{2} \varphi_{q}^{\text {ext }}(q)^{2} \mathrm{~d} q+ \\
& \int_{0}^{l} \varphi_{q}^{\text {vortex }}\left(q-q_{0}\right) \varphi_{q}^{\text {ext }}(q) \mathrm{d} q .
\end{aligned}
$$

Since we are only interested in the dependence of $U^{\operatorname{ext}}\left(q_{0}\right)$ on $q_{0}$, we can neglect the first two constant terms which are the magnetic energy of a vortex at rest and the energy of the external magnetic field in the junction, respectively. The last term, which is a convolution of the externally introduced phase gradient with the phase gradient profile of the vortex, is the potential energy corresponding to the magnetic dipole interaction.

The influence of the bias current $\gamma$ can be taken into account by adding a potential term corresponding to a constant driving force. This yields the total potential

$$
U\left(q_{0}\right)=\varphi_{q}^{\text {vortex }}\left(q-q_{0}\right) *_{q} \varphi_{q}^{\mathrm{ext}}(q)-2 \pi \gamma q_{0}
$$




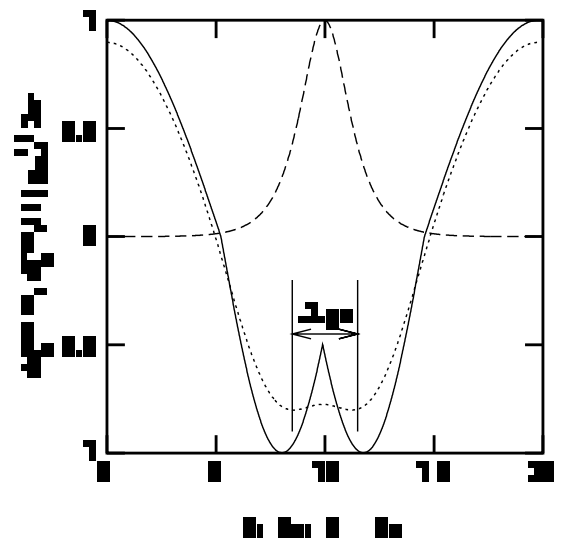

Figure 1.10: The phase gradient (solid line) $\varphi_{q}^{\text {ext }}(q)$ introduced by the external magnetic field, the phase gradient (long dashed) $\phi_{q}^{\text {vortex }}\left(q-q_{0}\right)$ associated with phase profile of a vortex (shifted to $q-q_{0}+10$ for visibility), and the effective potential (short dashed) $U\left(q_{0}\right)$, given by Eq. (1.18) with $\gamma=0$.

for the vortex, where $*_{q}$ denotes the convolution in $q$. The effect of the convolution of the externally introduced phase gradient with the magnetic profile of the vortex is indicated in Fig. 1.10. The phase gradient (solid line) is induced by a field in y-direction $(\Theta=\pi / 2)$, see heart shaped junction depicted in Fig. 1.9a. While the derivative of the phase gradient is discontinuous at $q=1 / 2 l$, the resulting potential is smooth at this point. Furthermore the separation $\Delta q_{0}$ between the minima and the height $U_{0}$ of the barrier are diminished by the convolution. In general, all local perturbations are smoothed to a length of the order of $\lambda_{J}$.

We now discuss how a specific geometry, like that of Fig. 1.9a, is related to the potential profile for an arbitrary field angle $\Theta$. The phase difference gradients correspond to shielding currents, which flow in the superconducting electrodes of the junction. Since these shielding currents are orthogonal to the magnetic field, the phase gradient along the junction is proportional to the scalar product of the normal vector of the junction with the external magnetic field. This is equivalent to the physical interpretation of the vortex magnetic moment interacting with the external magnetic field. Expanding the scalar product to its (orthogonal) components

$$
\varphi_{q}^{\mathrm{ext}}(q)=\vec{n}(q) \cdot \vec{h}=n_{x}(q) h_{x}+n_{y}(q) h_{y}
$$

yields an equation, in which the phase gradient is linear in each component of the field. Since also the convolution of the vortex magnetic moment with the external magnetic field is a linear operation, it is possible to separate the convolution for the calculation of the potential into two 
components:

$$
\begin{aligned}
\varphi_{q}^{\text {vortex }}\left(q-q_{0}\right) *_{q} \varphi_{q}^{\text {ext }}= & \varphi_{q}^{\text {vortex }}\left(q-q_{0}\right) *_{q} n_{x}(q) h_{x}+ \\
& \varphi_{q}^{\text {vortex }}\left(q-q_{0}\right) *_{q} n_{y}(q) h_{y} .
\end{aligned}
$$

We now abbreviate $\varphi_{q}^{\text {vortex }}\left(q-q_{0}\right) *_{q} n_{x}(q)$ by $U_{x}$, and substitute Eq. (1.20) into Eq. (1.18). This yields

$$
U\left(q_{0}\right)=U_{x}\left(q_{0}\right) h_{x}+U_{y}\left(q_{0}\right) h_{y}-2 \pi \gamma q_{0}
$$

as the total potential for the vortex motion.

We now return to the geometry in Fig. 1.9a. From the symmetry of the heart it can be seen immediately, that $\vec{n}_{y}(q)$ is symmetric with respect to $q=0$ and $q=l / 2$, while $\vec{n}_{x}(q)$ is antisymmetric with respect to these points. Therefore also $U_{y}\left(q_{0}\right)$ and $U_{x}\left(q_{0}\right)$ are symmetric and antisymmetric, respectively. Of special interest is the region at $q=l / 2$, since $U_{y}$ forms a double-well potential there, if the radius $R$ is large enough in relation to the Josephson length. The distance between the wells and the height of the barrier are strongly diminished with increasing Josephson length. At a certain critical value of the Josephson length the barrier ceases to exist for this geometry.

\subsubsection{Quantum mechanics of a vortex in a double well}

The Schrödinger equation for the center of mass motion of a vortex in normalized units is given by

$$
-i \hbar_{\text {norm }} \Psi=\hat{H} \Psi=(\hat{T}+\hat{U}) \Psi,
$$

where $\hat{U}$ denotes the potential energy operator, and

$$
\hat{T}=\frac{\partial}{\partial q^{2}} \frac{\hbar_{\text {norm }}^{2}}{16}
$$

corresponds to the kinetic energy operator for a particle of mass $m_{0}=8$. A numerical discretization and diagonalization of $\hat{H}$ yields the eigenstates, which are shown in Fig. 1.11a for $w=0.3 \mu \mathrm{m}, R=50 \mu \mathrm{m}, j_{c}=1000 \mathrm{~A} / \mathrm{cm}^{2}$.

The tunnel splitting between the two lowest energy eigenstates is proportional to the expected tunneling rate. The numerically calculated values for the tunnel splitting are shown as points in Fig.1.11b and tunneling rates calculated in WKB approximation are shown by the solid line. It can be seen, that the tunneling rate can be tuned within the experimentally accessible field-range by four orders of magnitude.

Applying a small $\mathrm{x}$-component lifts the degeneracy of the potential, since an antisymmetric potential component will be added. If this component is small, perturbation theory yields a correction for the energy eigenvalues of the uncoupled states inside each well:

$$
\Delta U=\left\langle\Psi\left|\hat{U}_{x}\right| \Psi\right\rangle h_{x} .
$$

Since $U_{x}\left(q_{0}\right)=-U_{x}\left(-q_{0}\right)$, and the states localized in the left/right well are symmetric, one state is shifted up, while the other state is shifted down in energy. 

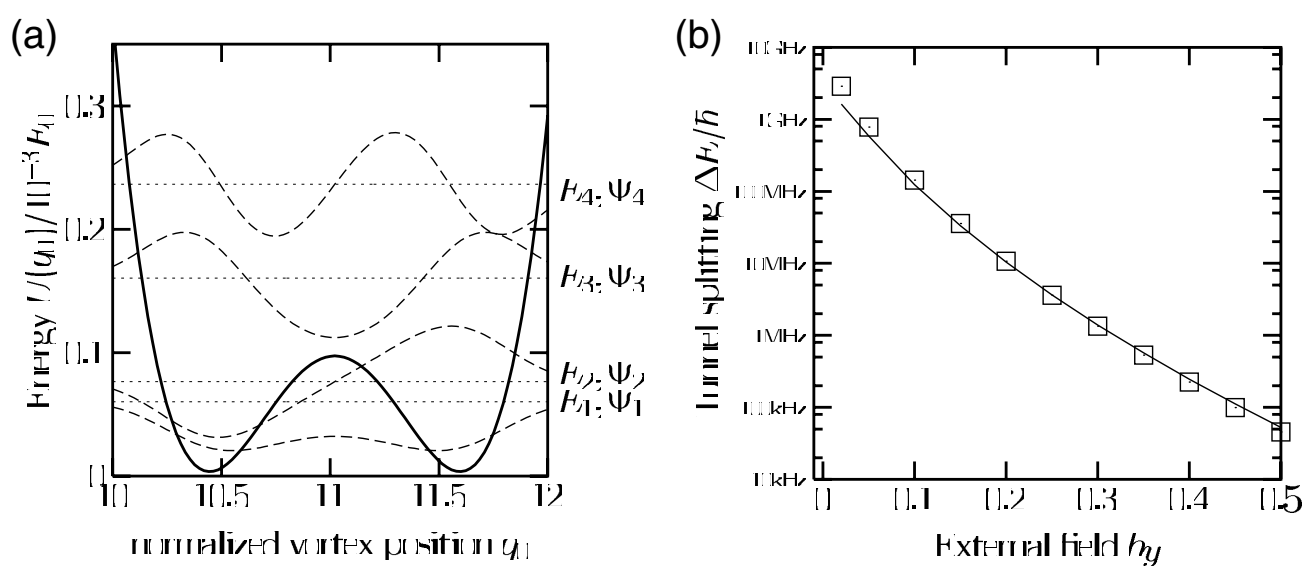

Figure 1.11: (a) Calculated lowest energy eigenstates for a junction of $w=0.3 \mu \mathrm{m}$. The energy levels are indicated by the dotted lines, the wave functions are indicated by the dashed lines.(b) Tunnel splitting between the states, according to the energy splitting of the lowest energy eigenstates.

At low temperatures, the vortex dynamics in the double-well potential is reduced to that of a two-state system, the Hamiltonian of which can be written as

$$
H=\Delta E\left(h_{y}\right) \sigma_{x}+\Delta U \sigma_{z}
$$

where $\Delta E$ is the overlap of the ground states, found by the WKB-method. It must be noted, that $\Delta E\left(h_{y}\right)$ depends exponentially on $h_{y}$ while $\Delta U$ depends only linearly on $h_{x}$. By controlling $h_{x}$ and $h_{y}$ it is possible to realize all single qubit operations.

\subsubsection{Depinning current and qubit readout}

For the experimental test of the readout procedure in the classical regime [47], we are interested in the depinning current of the vortex which is an experimentally accessible parameter. A vortex starts to move, when the pinning force due to the external field is compensated by the force exerted by the bias current. Calculating the derivative of Eq. (1.21) yields the force acting on the vortex

$$
F\left(q_{0}\right)=\frac{\partial U\left(q_{0}\right)}{\partial q_{0}}=\frac{\partial U_{x}\left(q_{0}\right)}{\partial q_{0}} h_{x}+\frac{\partial U_{y}\left(q_{0}\right)}{\partial q_{0}} h_{y}-2 \pi \gamma
$$

The equilibrium positions correspond to a zero net force. Using Eq. (1.21), we can write a condition for the equilibrium positions as

$$
F_{x}\left(q_{0}\right)\left(h_{x} / h\right)+F_{y}\left(q_{0}\right)\left(h_{y} / h\right)=2 \pi(\gamma / h),
$$

where $F_{x}$ denotes $\partial U_{x}\left(q_{0}\right) / \partial q_{0}$.

Solutions of Eq. (1.27) vanish, if local maxima/minima in the force are exceeded by $\gamma 2 \pi$. A vortex trapped in one of these stable equilibrium positions will be depinned at these currents. 


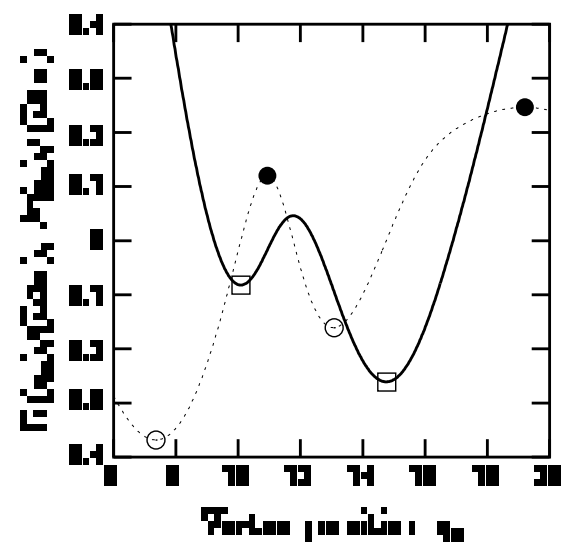

Figure 1.12: The minima of the potential $U\left(q_{0}\right)$ (solid line) are marked by open squares. The minima of the pinning force (dotted line) are marked by open circles, the maxima by solid circles.

The corresponding current is therefore called the depinning current $\gamma_{\mathrm{dep}, i}$, where $i$ is the index, if several stable positions exist. In Fig. 1.12, a potential without bias current is shown, together with the corresponding force. Each minimum of the potential, indicated by an open square, has one corresponding minimum (open circle) and maximum, (solid circle) of the force. For a given angle $\Theta$ of the external field, $\left(h_{x} / h\right)$ and $\left(h_{y} / h\right)$ in Eq. (1.27) are constant. Therefore the ratio $\rho_{\mathrm{dep}, i}=\left(\gamma_{\mathrm{dep}, i} / h\right)$ is constant for a specific angle of the field.

The dependence of $\rho_{\mathrm{dep}, i}$ on the angle of the external magnetic field can be found numerically. In Fig. 1.13, the dependence of $\rho_{\mathrm{dep}, i}$ on the field angle $\Theta$ is plotted by two lines for $i=1$, and $i=2$. We performed a test, if the vortex would be retrapped in the remaining stable positions. This happens if the spatial distance between the depinning position and the next maximum in the potential is so small, that the vortex does not gain enough kinetic energy to overcome this maximum. If the current is increased, the vortex is depinned from this remaining position at the corresponding depinning current. In this case we plotted the latter value of the depinning current in Fig. 1.13.

We carried out an experimental test of the preparation and readout scheme proposed above using the junction shown in Fig. 1.9b. Figure 1.13 shows the measured depinning current in dependence on the angle $\Theta$ of the external magnetic field. We measured the depinning current of the vortex after a clockwise and counterclockwise rotation as described in Ref. [47]. The two directions of rotation correspond to circles and squares in Fig. 1.13, respectively.

From the dependence of the depinning current on the angle the two ways of preparation can be associated with the numerically determined values of $\rho_{\text {dep }, i}$. At $\Theta=270^{\circ}$, we find numerically that $\rho_{\mathrm{dep}, 1}=\rho_{\mathrm{dep}, 2}$. This angle corresponds to an antisymmetric potential, which has a symmetric first derivative. Therefore the maximum values of the pinning force are identical. In Fig. 1.13 this theoretically predicted crossing is found in the experiment. The 


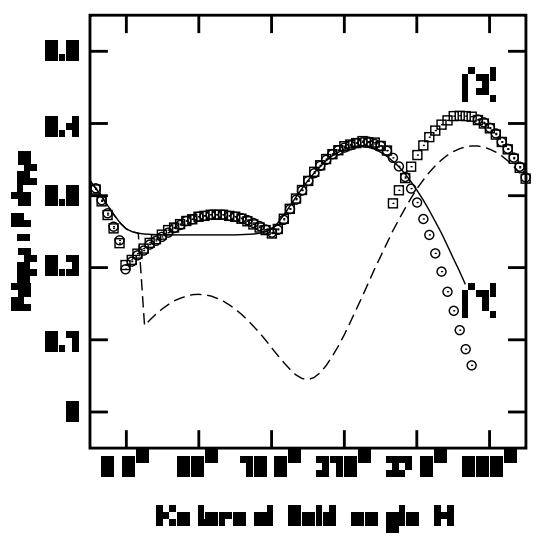

Figure 1.13: The normalized depinning current $\gamma_{\mathrm{dep}} / h$ plotted versus the angle $\Theta$ of the external field. Lines correspond to numerical calculation, squares (clockwise preparation of state, $\mathrm{i}=1$ ) and circles (counterclockwise preparation of state, $\mathrm{i}=2$ ) are the experimentally measured values.

pinning for state (2) is slightly higher than expected. This small discrepancy of $\rho_{\mathrm{dep}, i}$ indicates additional pinning possibly due to inhomogeneities, residual flux or geometrical reasons.

In the experiment, only at the readout angle $\Theta \approx 330^{\circ}$ the crossover between retrapping in state (2) and depinning from state (1) is observed as numerically predicted. The crossover from retrapping in state (1) to detection of state (2) shows a large discrepancy to the numerical prediction. We attribute the difference between the experimental and the numerical data in Fig. 1.13 in the range $\Theta \approx 45^{\circ} \ldots 160^{\circ}$ to the damping which has been neglected in the numerical calculation.

\subsection{Conclusions}

In our experiments, we have observed for the first time the quantum tunnelling of an individual vortex in a long Josephson junction [28]. We have also demonstrated the existence of well separated vortex energy levels in the potential well using microwave spectroscopy [28]. The dissociation of a bound vortex-antivortex pair, generated by magnetic field and bias current in a long junction, has been observed in the quantum regime [37]. These results are promising for using of Josephson vortices in long junctions as qubits for quantum information processing. The anharmonic oscillatory states of a vortex within a single well or, alternatively, the ground states of a vortex in a double well potential can be used as basis states for a qubit $[46,50]$. We have developed heart-shaped long junctions for use as qubits and have demonstrated a preparation and readout scheme for the vortex qubit in the classical regime [50] and thus verified the existence of bistable states. For the operation of the vortex qubit the width and 
height of the potential barrier and the symmetry of the double well are controlled by external fields. Macroscopic quantum coherence experiments using vortices in long sub-micron width junctions are currently in progress.

\section{Acknowledgments}

We gratefully acknowledge the contributions of C. Coqui, T. Duty, M. V. Fistul, Y. Koval, J. Lisenfeld, A. Lukashenko, B. A. Malomed to the work described in this chapter. We thank A. Abdumalikov, G. Blatter, Takeo Kato, G. Schön and S. Shnirman for fruitful discussions and IPHT Jena for the fabrication of one of the sets of samples used in this investigation. The partial financial support by Deutsche Forschungsgemeinschaft (DFG) and D-Wave Systems Inc. is acknowledged.

\section{Bibliography}

[1] Abo-Shaeer, J. R., Raman, C., Vogels, J. M., \& Ketterle, W. Observation of vortex lattices in Bose-Einstein condensates. Science 292, 476-479 (2001).

[2] Blaauwgeers, R., Eltsov, V. B., Krusius, M., Ruohio, J. J., Schanen, R., \& Volovik, G. E. Double-quantum vortex in superfluid ${ }^{3}$ He-A. Nature 404, 471-473 (2000).

[3] Huebener, R. P. Magnetic Flux Structures in Superconductors. Springer, (2001).

[4] Bugoslavsky, Y., Perkins, G. K., Qi, X., Cohen, L. F., \& Caplin, A. D. Vortex dynamics in superconducting $\mathrm{MgB}_{2}$ and prospects for applications. Nature 410, 563 - 565 (2001).

[5] Lee, C.-S., Janko, B., Derenyi, I., \& Barabesi, A.-L. Reducing the vortex density in superconductors using the ratchet effect. Nature 400, 337-340 (1999).

[6] Nori, F. and Savel'ev, S. Experimentally realizable devices for controlling the motion of magnetic flux quanta in anisotropic superconductors. Nature Materials 1, 179-184 (2002).

[7] Fazio, R. \& van der Zant, H. J. S. Quantum phase transitions and vortex dynamics in superconducting networks. Physics Reports 355, 235-334 (2001).

[8] Blatter, G., Feigel'man, M. V., Geshkenbein, A. I., \& Vinokur, V. M. Vortices in hightemperature superconductors. Rev. Mod. Phys. 66, 1125-1388 (1994).

[9] Tinkham, M. Introduction to Superconductivity. McGraw-Hill (1996).

[10] Clarke, J., Cleland, A. N., Devoret, M. H., Esteve, D., \& Martinis, J. M. Quantum mechanics of a macroscopic variable: The phase difference of a Josephson junction. Science 239, 992-997 (1988).

[11] Devoret, M. H., Esteve, D., Urbina, C., Martinis, J., Cleland, A., \& Clarke, J. Macroscopic quantum effects in the current-biased Josephson junction. In Quantum tunneling in condensed media. North-Holland (1992).

[12] Bennett, C. H. \& DiVincenzo, D. P. Quantum information and computation. Nature 404, 247-255 (2000).

[13] Nakamura, Y., Pashkin, Y. A., \& Tsai, J. S. Coherent control of macroscopic quantum states in a single-cooper-pair box. Nature 398, 786-788 (1999).

[14] Friedman, J. R., Patel, V., Chen, W., Tolpygo, S. K., \& Lukens, J. E. Quantum superposition of distinct macroscopic states. Nature 406, 43-46 (2000). 
[15] van der Wal, C. H., ter Haar, A. C. J., Wilhelm, F. K., Schouten, R. N., Harmans, C. J. P. M., Orlando, T. P., Lloyd, S., \& Mooij, J. E. Quantum superposition of macroscopic persistent-current states. Science 290, 773-777 (2000).

[16] Pashkin, Y. A., Yamamoto, T., Astafiev, O., Nakamura, Y., Averin, D., \& Tsai, J. S. Quantum oscillations in two coupled charge qubits. Nature 421, 823-826 (2003).

[17] Chiorescu, I., Nakmura, Y., Harmans, C. J. P. M., \& Mooij, J. E. Coherent quantum dynamics of a superconducting flux qubit. Science 299, 1869-1871 (2003).

[18] Yamamoto, T., Pashkin, Y. A., Astafiev, O., Nakamura, Y., \& Tsai, J. S. Demonstration of conditional gate operation using superconducting charge qubits. Nature 425, 941-944 (2003).

[19] Wallraff, A., Schuster, D. I., Blais, A., Frunzio, L., Huang, R.-S., Majer, J., Kumar, S., Girvin, S. M., \& Schoelkopf, R. J. Circuit quantum electrodynamics: Coherent coupling of a single photon to a Cooper pair box. Nature 431, 162 (2004).

[20] Chiorescu, I., Bertet, P., Semba, K., Nakamura, Y., Harmans, C. J. P. M., \& Mooij, J. E. Coherent dynamics of a flux qubit coupled to a harmonic oscillator. Nature 431, 159-162, September (2004).

[21] Hoekstra, H. F. T., Griessen, R., Testa, M. A., Brinkmann, M., Westerholt, K., Kwok, W. K., \& Crabtree, G. W. General features of quantum creep in high- $t_{c}$ superconductors. Phys. Rev. Lett. 80, 4293-4296 (1998).

[22] Nicodemi, M. \& Jensen, H. J. Creep of superconducting vortices in the limit of vanishing temperature: A fingerprint of off-equilibrium dynamics. Phys. Rev. Lett. 86, 4378-4381 (2001).

[23] van der Zant, H. S. J., Fritschy, J. F. C., Orlando, T. P., \& Mooij, J. E. Dynamics of vortices in underdamped josephson-junction arrays. Phys. Rev. Lett. 66, 2531-2534 (1991).

[24] Tighe, T. S., Johnson, A. T., \& Tinkham, M. Vortex motion in two-dimensional arrays of small, underdamped josephson junctions. Phys. Rev. B 44, 10286-10290 (1991).

[25] Ustinov, A. V. Solitons in Josephson junctions. Physica D 123, 315-329 (1998).

[26] Kato, T. \& Imada, M. Macroscopic quantum tunneling of a fluxon in a long Josephson junction. J. Phys. Soc. Jpn. 65(9), 2963-2975 (1996).

[27] Shnirman, A., Ben-Jacob, E., \& Malomed, B. A. Tunneling and resonant tunneling of fluxons in a long Josephson junction. Phys. Rev. B 56(22), 14677-14685 (1997).

[28] Wallraff, A., Lisenfeld, J., Lukashenko, A., Kemp, A., Fistul, M., Koval, Y., \& Ustinov, A. V. Quantum dynamics of a single vortex. Nature 425, 155 (2003).

[29] Koval, Y., Wallraff, A., Fistul, M., Thyssen, N., Kohlstedt, H., \& Ustinov, A. V. Narrow long Josephson junctions. IEEE Trans. Appl. Supercond. 9, 3957-3961 (1999).

[30] McLaughlin, D. W. \& Scott, A. C. Perturbation analysis of fluxon dynamics. Phys. Rev. A 18, 1652-1980 (1978).

[31] Grønbech-Jensen, N., Lomdahl, P., \& Samuelsen, M. Phase locking of long annular Josephson junctions coupled to an external rf magnetic field. Phys. Lett. A 154(1,2), 14-18 (1991).

[32] Ustinov, A. V. \& Thyssen, N. Experimental study of fluxon dynamics in a harmonic potential well. J. of Low Temp. Phys. 106, 193-200 (1997). 
[33] Fulton, T. A. \& Dunkleberger, L. N. Lifetime of the zero-voltage state in Josephson tunnel junctions. Phys. Rev. B 9, 4760-4768 (1974).

[34] Wallraff, A., Lukashenko, A., Coqui, C., Kemp, A., Duty, T., \& Ustinov, A. V. Switching current measurements of large area Josephson tunnel junctions. Rev. Sci. Instrum. 74, 3740 (2003).

[35] Wallraff, A., Duty, T., Lukashenko, A., \& Ustinov, A. V. Multi-photon transitions between energy levels in a current-biased Josephson tunnel junction. Phys. Rev. Lett. 90, 037003 (2003).

[36] Martucciello, N., Mygind, J., Koshelets, V. P., Shchukin, A., Filippenko, L., \& Monaco, R. Fluxon dynamics in long annular Josephson tunnel junctions. Phys. Rev. B 57(9), 5444-5449 (1998).

[37] Fistul, M. V., Wallraff, A., Koval, Y., Lukashenko, A., Malomed, B. A., \& Ustinov, A. V. Quantum dissociation of a vortex-antivortex pair in a long josephson junction. Phys. Rev. Lett. 91, 257004 (2003).

[38] Martucciello, N. \& Monaco, R. Static properties of annular Josephson tunnel junctions. Phys. Rev. B 54(13), 9050 (1996).

[39] Maki, K. Phys. Rev. Lett. 39, 46 (1977).

[40] Krive, I. V., Malomed, B. A., \& Rozhavsky, A. S. Phys. Rev. B 42, 273 (1990).

[41] Kato, T. Dimensional crossover by a local inhomogeneity in soliton-pair nucleation. $J$. Phys. Soc. Jpn. 69, 2735 (200).

[42] A. Kemp, M.V. Fistul, A. Wallraff, Y. Koval, A. Lukashenko, B.A. Malomed, \& A.V. Ustinov Energy Level Spectroscopy of a Bound Vortex-Antivortex Pair to be published in: P. Delsing, C. Granata, Y. Pashkin, B. Ruggiero and P. Silvestrini Quantum Computation: solid state systems, Kluwer Academic Plenum Publishers, to be published on December 2004.

[43] Martinis, J. M., Nam, S., Aumentado, J., \& Urbina, C. Rabi oscillations in a large Josephson-junction qubit. Phys. Rev. Lett. 89, 117901 (2002).

[44] Vion, D., Aassime, A., Cottet, A., Joyez, P., Pothier, H., Urbina, C., Esteve, D., \& Devoret, M. H. Manipulating the quantum state of an electrical circuit. Science 296, 886-889 (2002).

[45] Yu, Y., Han, S., Chu, X., Chu, S.-I., \& Wang, Y. Coherent temporal oscillations of macroscopic quantum states in a Josephson junction. Science 296, 889-892 (2002).

[46] Wallraff, A., Koval, Y., Levitchev, M., Fistul, M. V., \& Ustinov, A. V. Annular long Josephson junctions in a magnetic field: Engineering and probing the fluxon potential. J. Low Temp. Phys. 118(5/6), 543-553 (2000).

[47] Kemp, A., Wallraff, A., \& Ustinov, A. V. Testing a state preparation and read-out protocol for the vortex qubit. Physica $C$ 368, 324 (2002).

[48] Ustinov, A. V., Malomed, B. A., \& Thyssen, N. Soliton trapping in a harmonic potential: experiment. Phys. Lett. A 233, 239 (1997).

[49] Kemp, A. Fluxon states in heart-shaped Josephson junction. Master's thesis, Physikalisches Institut III, Universität Erlangen-Nürnberg, (2001).

[50] Kemp, A., Wallraff, A., \& Ustinov, A. V. Josephson vortex qubit: Design, preparation and read-out. Phys. Stat. Sol. (b) 233(3), 472-481 (2002). 